\title{
Medical Monitoring and Management System of Mobile Thyroid Surgery Based on Internet of Things and Cloud Computing
}

\author{
Heng Kong $\mathbb{D}^{1}$ and Jixin Chen ${ }^{2}$ \\ ${ }^{1}$ Department of Thyroid and Breast Surgery, Baoan Central Hospital of Shenzhen, The Fifth Affiliated Hospital of \\ Shenzhen University, Shenzhen, 518102 Guangdong, China \\ ${ }^{2}$ Department of General Surgery, Shenzhen University General Hospital, Shenzhen University Clinical Medical Academy, Shenzhen, \\ 518000 Guangdong, China
}

Correspondence should be addressed to Heng Kong; generaldoc@126.com

Received 13 April 2021; Revised 11 May 2021; Accepted 4 June 2021; Published 21 June 2021

Academic Editor: Wenqing Wu

Copyright (c) 2021 Heng Kong and Jixin Chen. This is an open access article distributed under the Creative Commons Attribution License, which permits unrestricted use, distribution, and reproduction in any medium, provided the original work is properly cited.

\begin{abstract}
With the rapid development of the Internet of Things and cloud computing technologies, the Internet of Things technology based on comprehensive perception and interconnection and cloud computing based on virtualization, dynamic resources, and parallel computing have become the driving force for the innovation and development of informatization and intelligence. The cloud-based Internet of Things mobile medical is an ecosystem of health information and medical information, with the medical Internet of Things at its core and highly mobile and highly shared information. Therefore, in the context of in-depth research on mobile medical care, research on mobile postoperative thyroid monitoring and management systems based on the Internet of Things and cloud computing is a practical tool for promoting the development of mobile medical systems. Monitoring and Management. In this experiment, 48 cases of patients undergoing thyroid surgery were selected from a hospital. The experimental group was informed by the experiment that they need to be equipped with sensors. The control panel and GPS positioning module are used to obtain the exact position of the patient at the first time. The loading of the sensor is agreed by the patient and the patient's family. Afterward, the control group will not be processed. It will conduct functional tests and software performance tests on the mobile medical monitoring and management system and analyze the satisfaction of medical staff with the mobile medical monitoring and management system. Experiments have proved that the cloud computing medical monitoring and management system is used to obtain the exact location of the patient in the first time, and the response time needs to be shorter. The response time of the system increases with the increase of the number of sensors $(P<0.05)$, which shows that the mobile medical monitoring and management system is essential for medical care and medical care. Obtaining the exact position of the patient for the first time is of great importance for the successful rescue of the patient.
\end{abstract}

\section{Introduction}

With the continuous development of the Internet of Things, cloud computing, and smart hardware technologies, and the continuous popularization of mobile medical monitoring equipment, more people tend to use mobile smart terminals to obtain services. It can be seen from this that the products of the information age such as big data and cloud computing have had a large impact on the architecture of traditional medical systems. On this basis, the state proposed medical information as an important direction for the development of medical physique reform. With the trend and encouragement of national policies, more and more service providers can provide medical services, allowing increasingly people to access high-quality medical and health services.

With the development of modern society and economy, the quality of life has gradually improved. People have realized the importance of health and daily maintenance. People have gradually paid attention to physical exercise, improved dietary structure, and paid attention to daily health data. Patients no longer want to be limited to the traditional mode of registering a doctor. People hope that medical services can 
be provided through multiple channels and multiple medical modes. Mobile medical monitoring can share the hospital's original information system with greater mobility and flexibility. The mobile application can simplify the work process and improve the overall work efficiency. Another advantage of mobile applications is that it can reduce medical errors and can effectively reduce information asymmetry in the implementation of nursing handover, improper time management, patient information collection errors, or patient care errors.

After half a century of exploration in the field of mobile medical monitoring at home and abroad, many achievements have been made in sensors, damage identification, safety assessment, and system integration. Edafe et al. reviewed the current wearable mobile medical monitoring system, focusing on textile and wireless sensor networkbased devices. These monitoring systems are composed of various types of small physiological sensors, transmission modules, and processing capabilities. In addition, the wearable mobile medical monitoring system has the potential to change the medical model by providing inexpensive, wearable, and unobtrusive solutions for continuous medical health monitoring. It is hoped that the current survey can provide guidance for future research improvements and provide reference for current achievements and maturity. These systems can also be used as an effective method to identify the technical advantages and disadvantages of the current state of the art in wearable device solutions. However, the experimental results lack more data support so that the wearable mobile medical monitoring system cannot be convincing $[1,2]$. von Ahnen et al. proposed a self-learning scheme for patient activity recognition. In this scheme, the patient only needs to carry an ordinary smartphone containing ordinary motion sensors. After collecting data in real-time through a smartphone, we use coordinate system transformation to preprocess the data to eliminate the influence of the phone's orientation. Then extract a set of robust and effective features from the preprocessed data. Since patients may inevitably perform various unpredictable activities without prior knowledge in the training data set, we propose a selflearning activity recognition scheme. This program determines whether there are prior training samples and labeled categories in the training pool. These samples match the unpredictable activity data well. However, because ordinary smartphones often forget to bring data, the data is not comprehensive. The self-learning activity recognition program does not immature [3]. Mchenry explained the research status of the medical monitoring systems from the aspects of mobile medical treatment, physiological index testing equipment, medical institution-level monitoring system, home monitoring system, etc. From the three aspects of health monitoring cloud platform, wearable medical equipment, and human sensor network, the research hotspots of medical monitoring system are analyzed. Pointed out the problems of the medical monitoring system based on the Internet of Things and predicted its future development. However, his research did not clearly propose how to solve the problem of the medical monitoring system, and the overall research lacks data support $[4,5]$.
This article is to study the medical monitoring and management system after mobile thyroid surgery. On the basis of summarizing the existing Internet of Things and cloud computing, a mobile medical monitoring and management system is established. The basic physiological parameters of patients after thyroid surgery are collected through sensors, and these basic parameters are collected. The normal parameters are preprocessed, then, this processed data is entered into the cloud computing platform via the wireless sensor network, and the game data is analyzed and processed on the cloud computing platform. Use cloud computing technology to compare stored medical records and medical information, find similar physiological parameters from large amounts of data to realize early warning of disease progression assess and monitor risks, and help users create a differentiated medical service mechanism.

\section{Mobile Medical Monitoring and Management System Based on Internet of Things and Cloud Computing}

\subsection{Conceptual Design of Each Functional Sublayer of the System}

2.1.1. Field Sensor Network. The design of the cloud computing system should fully consider the future development. It will be used as a basic platform for the deployment of related software, rather than a simple management system. The subsequent research and development upgrades of users or enterprises will be carried out on the basis of this platform. In the technical architecture, advanced application platforms and development platforms must be adopted, and each relevant application function system needs to realize the corresponding specific business functions [6, 7]. Serviceoriented, component-oriented technical frameworks and concepts should be adopted between systems, which is conducive to future business formulation or reorganization and development, and at the same time, facilitates service management. The system must have a certain degree of selfadaptability to prepare for future changes in the environment and requirements. Scalability is mainly reflected in that the system should be easy to expand and upgrade, and the scalability of the system can be improved by adopting distributed design, modular design of the system structure $[6,8]$. This system should fully consider the changes in user visits and network environment and make adjustments and optimizations in the physical architecture at the right time. Since this system is positioned as the basic platform of structural health monitoring, it must meet the requirements of system target changes. The schematic diagram of the overall system scheme is shown in Figure 1.

At present, the development direction of wireless sensors is to have both data collection and analysis functions. However, due to the limitation of power consumption of wireless sensors, its analysis and processing functions are relatively simple and inconvenient to upgrade. After constructing the structural health monitoring Internet of Things, the function of the sensor should be relatively simple, that is, to sense the monitored amount and transmit it upwards $[9,10]$. There are 


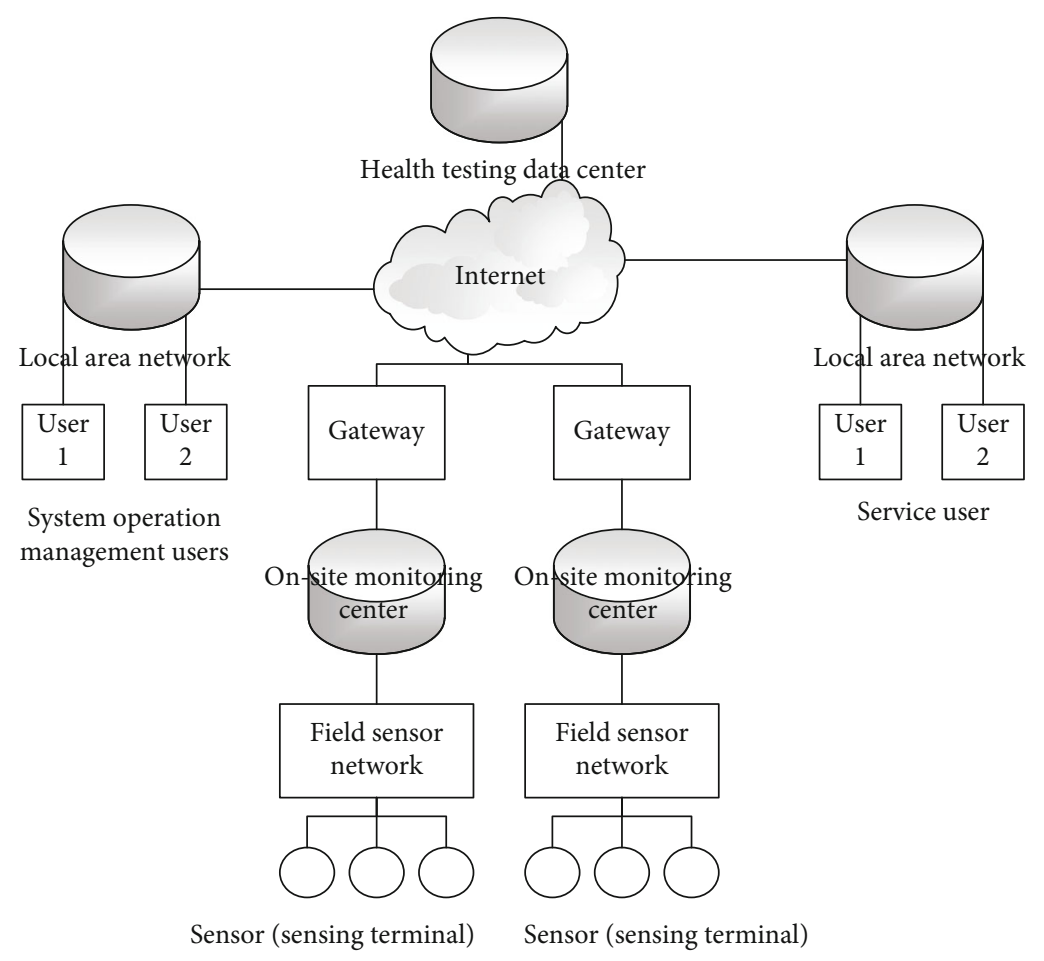

Figure 1: Schematic diagram of the overall system scheme.

a variety of short-range wired and wireless networking technologies, and different structures can use appropriate networking technologies to build on-site sensor networks according to specific design requirements, mainly considering the amount of monitoring, network coverage, network capacity, transmission rate, and security reliability and economy are required. No matter what kind of networking technology is adopted, the relevant information must eventually be gathered to the on-site monitoring center, so that the information can be transmitted to the data center; at the same time, the on-site monitoring center must also meet the control of each sensor node $[11,12]$. At present, the typical networking technology of wireless sensor networks is ZigBee network. In terms of wired sensor networks, fieldbus is a more typical networking technology with more applications.

2.1.2. Management System Architecture Design. The cloud computing platform system application is composed of multiple functional parts. To facilitate the operation, management, and maintenance of the system, the solution has designed a complete and visualized management subsystem for the operation and maintenance personnel to ensure the normal operation of the system, and at the same time, it is convenient for users to connect and maintain use resources in the cloud $[13,14]$. The economics of the system means that the revenue of the system is greater than the total cost of expenditure. Among them, the cloud platform system expenditure is mainly composed of operation and maintenance costs and investment costs for development, and the system application design should provide users with corre- sponding economic benefits $[15,16]$. In the process of system design and development, some open source software and technologies should be selected to reduce development costs. Practicability requirements meet the operability and user friendliness of the cloud platform system, provide users with convenient and reliable services, include whether the system platform can operate normally after the completion of the construction, and provide safety guarantees for real-time monitoring and safety assessment of the structure $[17,18]$. System management users interact with the data center database server through the C/S mode, and external service users access the WEB server through the $\mathrm{B} / \mathrm{S}$ mode to interact with the data center database server, as shown in Figure 2.

\subsection{Vibration Signal Preprocessing}

2.2.1. Eliminate Trend Items. Assuming that the sampled data received by the structural health monitoring cloud is a digital signal after calibration conversion, the calibration conversion is completed by the field data acquisition base station, and the deviation from the baseline contained in the signal is a trend item of the signal, which needs to be removed $[18,19]$. It is a more common method to use the least square method to eliminate the trend item:

$$
Y(t)=X(t)-Z(t)
$$

In the formula, $X(t)$ is to read the sampled data, the polynomial function fits $X(t)$ to obtain the polynomial coefficient vector $a$ of the trend term, and the estimated value $Z(t)$ of the polynomial trend term at each sampling point determined by 


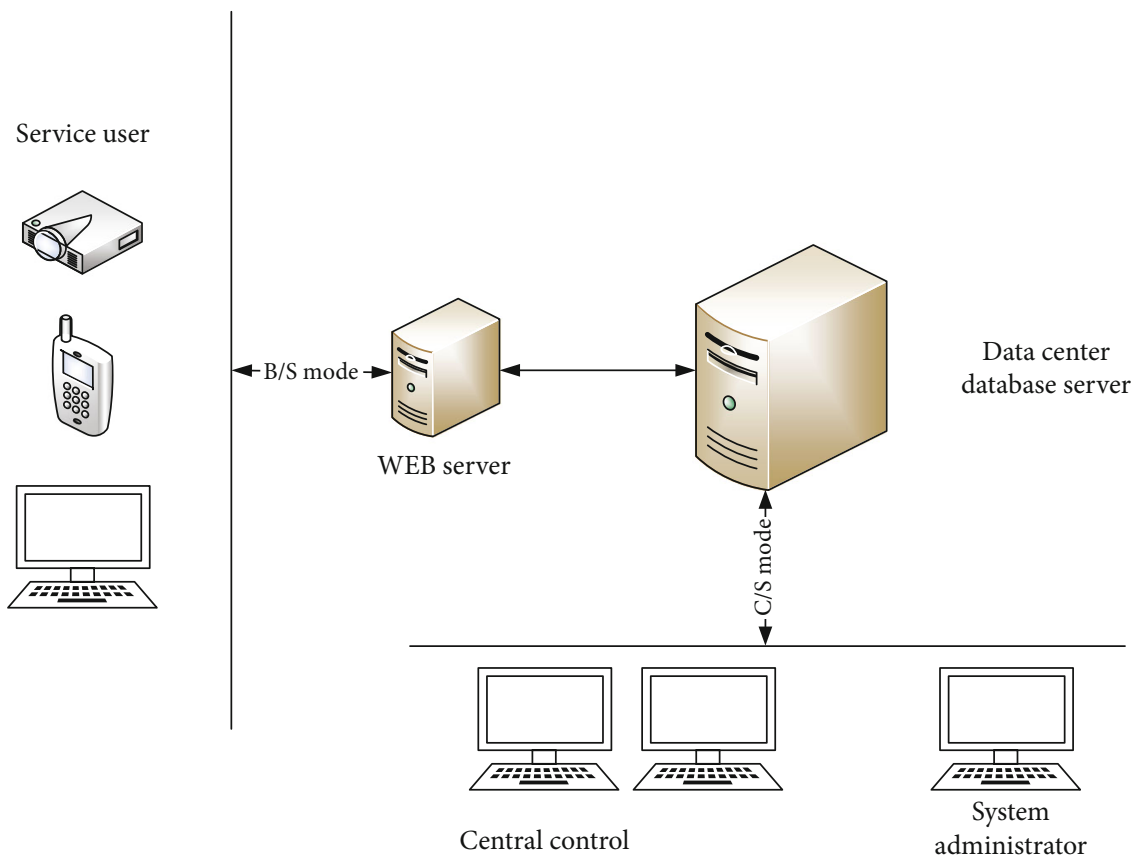

Figure 2: $\mathrm{C} / \mathrm{S}$ and $\mathrm{B} / \mathrm{S}$ hybrid architecture of medical monitoring management system.

a. In the processing flow, through coarse-grained correlation based on the analysis, it is obvious that the adjacent steps are flow-related and cannot be executed in parallel within the task.

2.2.2. Smoothing. The vibration signals collected by the health monitoring system are generally mixed with noise components and are affected by high-frequency component noise:

$$
P\left(y_{i} \mid X_{i}\right)=\pi\left(X_{i}\right)^{y_{i}}\left[1-\pi\left(X_{i}\right)\right]^{1-y_{i}}
$$

Here, $X_{i}=\left[x_{i 1}, x_{i 2}, \cdots, x_{\text {in }}\right] \quad i=1,2, \cdots, n$, the maximum likelihood function of $n$ sample observations is

$$
L(\beta \mid X, y)=\prod_{i=1}^{n}\left[\pi\left(X_{i}\right)\right]^{y_{i}}\left[1-\pi\left(X_{i}\right)\right]^{1-y_{i}}
$$

The log-likelihood function is

$$
l(\beta)=\sum_{i=1}^{n}\left\{y_{i} \ln \left[\pi\left(X_{i}\right)\right]+\left(1-y_{i}\right)\left[1-\pi\left(X_{i}\right)\right]\right\} .
$$

Too many smoothing times will significantly reduce the peak value of the spectrum curve, make the body shape wider, and even lead to larger recognition errors.

2.2.3. Infinite Impulse Response IIR Digital Filter. The purpose of filtering time-domain method and frequencydomain methods is to achieve signal frequency selection, filter out designated frequency components, and retain the required frequency components [20]. The filtering expres- sion of the IIR filter can be defined as the following difference equation:

$$
y(n)=\sum_{k=0}^{M} a_{k} x(n-k)-\sum_{k=1}^{N} b_{k} y(n-k)
$$

In the formula, $x(n)$ is the input time-domain signal sequence; $y(n)$ is the output time-domain signal sequence; $a_{k}, b_{k}$ is the filter coefficient; $M$ is the number of zeros of the filter system transfer function; $N$ is the filter order. The IIR filter not only inputs the finite term of the original signal for calculation but also considers the output term before the filter. Clearly, the calculation and execution of the filter are realized through the loop body, and each loop outputs a filtered value. The output result of the previous loop needs to be used as the input of the subsequent loop, and the loop has relevance $[21,22]$.

\subsubsection{Time-Frequency Domain Processing of Vibration Signals}

(1) Time Domain Processing. The random vibration signal has no obvious regularity. Let $\{x(k)\}$ be the discrete data sequence of the random vibration signal, the length is $N$, and the sampling time step is $\Delta t$. The mean value of the random vibration signal is estimated as:

$$
\mu_{x}=\frac{1}{N} \sum_{k=1}^{N} x(k)
$$

For this kind of averaging, the original data segment can be divided into several subdata segments of equal length to 
obtain the average value, respectively, and then the calculation results of the subdata segments are summed and averaged to obtain the average value estimate. The processes of averaging the data segments are not related and can be executed in parallel, and then the results of the parallel execution are summarized to obtain the final result. For the mean square estimate:

$$
\psi_{x}^{2}=\frac{1}{N} \sum_{k=1}^{N} x^{2}(k)
$$

The calculation process is the same as the mean estimation. Variance estimation:

$$
\sigma_{x}^{2}=\frac{1}{N} \sum_{k=1}^{N}\left[x(k)-\mu_{x}\right]^{2}=\psi_{x}^{2}-\mu_{x}^{2} .
$$

The calculation process can be decomposed into two unrelated subtasks to calculate the mean and mean square estimation. The parallelism of its task is embodied in that it can be decomposed into subtasks that can be executed in parallel, and its expression is

$$
R_{x x}(k)=\frac{1}{N} \sum_{i=1}^{N-k} x(i) x(i+k) .
$$

It can be seen from Expression (9) that the calculations of the instantaneous function values of the autocorrelation function are not related and can be executed in parallel. Since the calculation of the autocorrelation function also takes the single-channel random vibration time-domain signal as the input, the effect of parallel calculation on the calculation efficiency is not considered here.

$$
R_{x y}(k)=\frac{1}{N-k} \sum_{i=1}^{N-k} x(i) y(i+k) .
$$

The calculation of the cross-correlation function is also a two-level nested loop body calculation task, but its input signal is sampled data from two different channels, and the rest is the same as the calculation of the autocorrelation function [23].

In the structural health monitoring system, some physical quantities sometimes need to be acquired by transforming other physical quantities. For example, the speed signal or displacement signal can be obtained by the acceleration signal (twice) integration. The calculation and evaluation formula is

$$
y(k)=\Delta t \sum_{i=1}^{k} \frac{x(i-1)+x(i)}{2} .
$$

The calculation of the value of each discrete point of the signal after integration is not related and can be calculated in parallel. Since its input is a time series, parallelism here is of little significance to distributed computing, and it can only be used to increase the running speed of the time-domain integral calculation within the node.

(2) Frequency Domain Processing. Ultrasound images have high requirements for edge detail and are nonstationary signals that cannot be met by traditional Fourier transformbased signal denoising methods. Ultrasonic speckle suppression and denoising methods can be broadly divided into spatial area local statistical filtering, anisotropic diffusion filtering, and wavelet transform-based filtering. The autopower spectral density function is the Fourier transform of the autocorrelation function, namely,

$$
\begin{gathered}
S_{x x}(k)=\frac{1}{N} \sum_{n=0}^{N-1} R_{x x}(n) e^{-i 2 \pi k n / N}, \\
S_{x y}(k)=\frac{1}{N} \sum_{n=0}^{N-1} R_{x y}(n) e^{-i 2 \pi k n / N} .
\end{gathered}
$$

The power spectral density function can be used as the input data of some specific modal parameter identification methods, namely,

$$
\begin{gathered}
H(k)=\frac{S_{x y}(k)}{S_{x x}(k)}, \\
C_{x y}(k)=\frac{\left|S_{x y}(k)\right|^{2}}{S_{x x}(k) S_{y y}(k)} .
\end{gathered}
$$

In the formula, $S_{x x}(k)$ means self-power spectrum estimation of random vibration excitation signal; $S_{x y}(k)$ means cross-power spectrum estimation of random vibration excitation and response signal. The left is used for the coherence function to evaluate the quality of the frequency response function estimation result $[24,25]$.

\section{Experimental Design of Mobile Medical Monitoring and Management System}

3.1. Test Subject. In this trial, 48 patients undergoing thyroid surgery in a hospital from 2019 to 2020 were selected and randomly divided into two groups with 24 people in each group. The experimental group was informed by the experiments that it needed to be equipped with sensors and used the control panel and GPS positioning module for the first time. Obtain the exact location of the patient at a time, and load the sensor with the consent of the patient and the patient's family; the control group will not be processed. The general information of these 48 patients is presented in Table 1. For functional tests and software performance tests of the mobile medical monitoring and management system, and at the same time, analyzing the satisfaction of the medical staff with the mobile medical monitoring and management system, the research tool used in the questionnaire survey method is the questionnaire, which uses the Likert 
TABLE 1: General information of the two groups of patients.

\begin{tabular}{|c|c|c|c|c|c|}
\hline \multirow{2}{*}{ Group } & \multicolumn{2}{|c|}{ Number of cases } & \multirow{2}{*}{ Years } & \multirow{2}{*}{ Course of disease } & \multirow{2}{*}{ Years of education } \\
\hline & Male & Female & & & \\
\hline Experiment group & 16 & 8 & $43.27 \pm 11.21$ & $7 \pm 5.5$ & $8 \pm 5.12$ \\
\hline Control group & 14 & 10 & $41.98 \pm 14.02$ & $9 \pm 3.4$ & $9 \pm 4.57$ \\
\hline
\end{tabular}

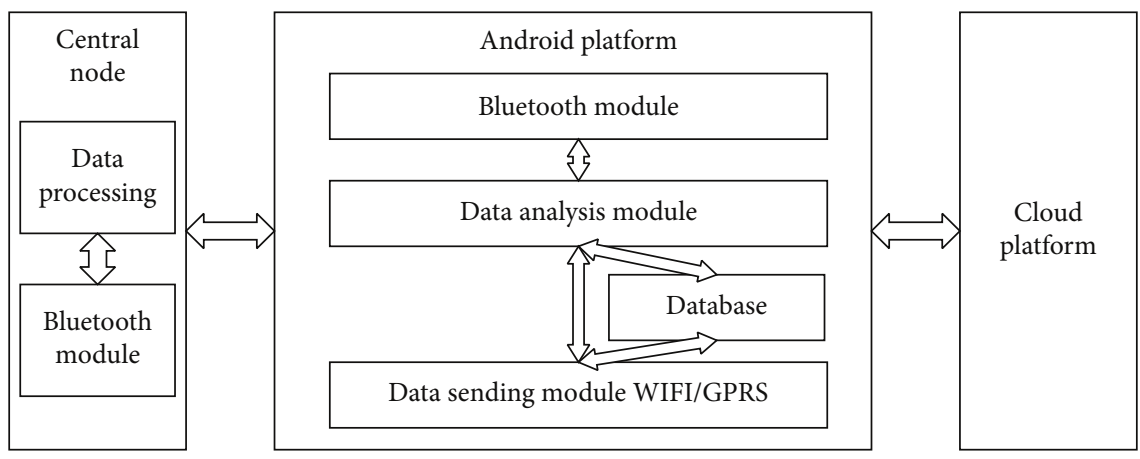

FIgURE 3: System structure frame diagram.

TABLE 2: System function test table.

\begin{tabular}{|c|c|c|c|}
\hline Functional module & Function name & Test steps & Test results \\
\hline \multirow[t]{4}{*}{ Login/registration module } & Login & Perform login operation & Successfully logged in \\
\hline & Control panel & Manually control other module operations & Test success \\
\hline & GPS positioning module & Manual GPS positioning operation & Test success \\
\hline & Bluetooth module & Connection between central nodes & Test success \\
\hline \multirow[t]{4}{*}{ Main functions of the system } & Data analysis module & Cloud platform for data analysis operations & Test success \\
\hline & Data storage module & Database for data storage operations & Test success \\
\hline & Data sending module & Cloud platform for data sending operations & Test success \\
\hline & System home & Enter the system homepage operation & Test success \\
\hline
\end{tabular}

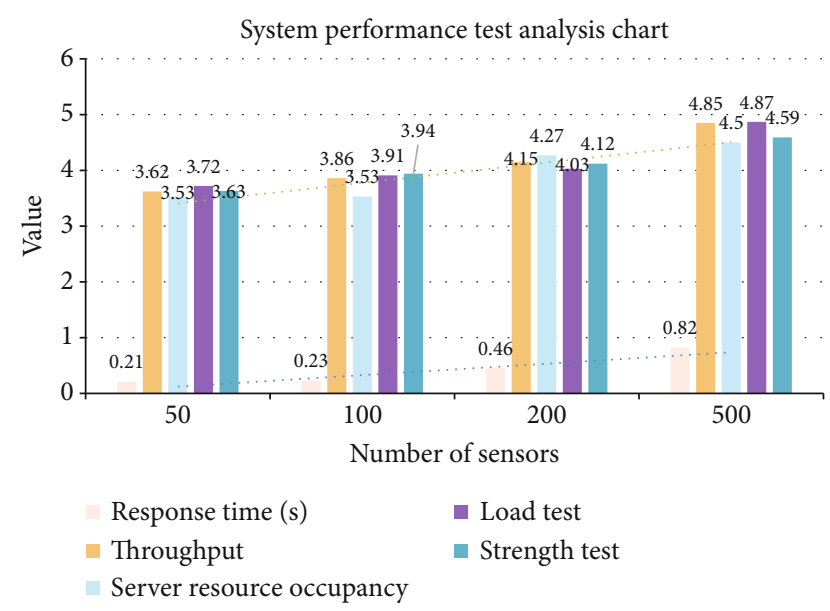

FIGURE 4: System performance test analysis chart.

five-level scoring method, the score ranges from 1 to 5, which, respectively, represent completely disagree with this view, disagree with this view, neither disagree nor agree, agree with it view, and I fully agree with that view, respec- tively. The higher the price, the more you agree with the point of view.

\subsection{Experimental Method}

3.2.1. Cloud Computing Medical Monitoring and Management System. In the daily work of thyroid postoperative care, the medical monitoring and management system uses the Internet of Things and cloud computing technology, and uses real-time monitoring procedures for the physiological indicators in the patient care process, and uploads and transmits information in time, and the members of the nursing team conduct real-time monitoring feedback, etc. Through the Internet of Things technology to implement all-weather monitoring of each patient and each hospital bed, to achieve a 24-hour uninterrupted effect, so that the nursing team members can know the patient's infusion situation at any time in the nurse station or mobile state and carry out timely replacement of infusion bottles and handling of accidental infusions improve the work efficiency of medical staff and bring better experience to patients [26, 27].

3.2.2. System Composition. The medical monitoring and management system consists of monitoring instruments, 
TABle 3: Monitoring system energy consumption data sheet.

\begin{tabular}{lcccc}
\hline & Power consumption & Equipment cost & System standby time & Scope of action \\
\hline Surveillance system & Medium & High & Long & Small \\
Medical monitoring and management system & Cheap & Cheap & Long & Wide \\
\hline
\end{tabular}

TABLE 4: Monitoring system transmission speed data table.

\begin{tabular}{|c|c|c|c|c|c|}
\hline & $\begin{array}{c}\text { Data transmission } \\
\text { speed }\end{array}$ & Accuracy & $\begin{array}{l}\text { Information processing } \\
\text { capability }\end{array}$ & $\begin{array}{l}\text { Helpful to medical } \\
\text { staff }\end{array}$ & $\begin{array}{c}\text { Anti- } \\
\text { interference }\end{array}$ \\
\hline Surveillance system & Slow & Medium & Medium & Medium & Medium \\
\hline $\begin{array}{l}\text { Medical monitoring and management } \\
\text { system }\end{array}$ & Fast & Accurate & Power & Power & Power \\
\hline
\end{tabular}

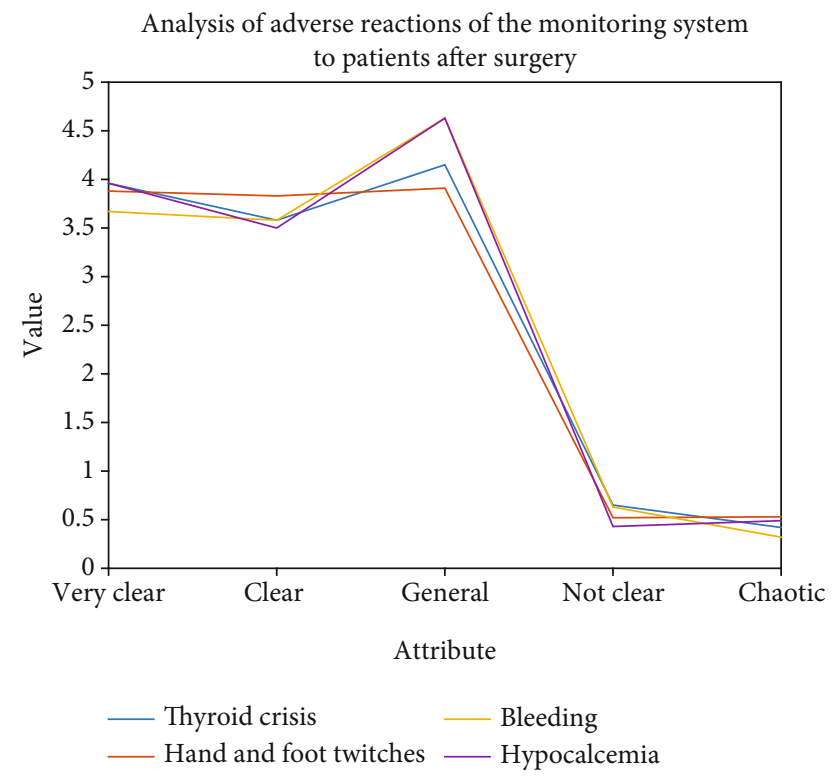

FIGURE 5: Analysis of adverse reactions of the monitoring system to patient surgery.

data receivers, and computer screen (with network interface), and its structural framework is shown in Figure 3. First, the monitoring instrument is used to collect data on various physiological indicators of the patient's body, and then the relevant information is uploaded and sent to the data receiver. Each bed in the room is equipped with a monitor and hung with a hook under the screen window. Each information bar is equipped with a data receiver, and the monitor will send data. In the cloud service layer, the data uploaded through the transmission layer is stored in a database cluster, and the data processing component analyzes the data according to related algorithms and organizes related health files or related medical records [28-30].

3.3. Establish Model Evaluation Index System. The evaluation index is a specific evaluation item determined according to some evaluation goals, which can reflect some basic characteristics of the evaluation object. The index is specific and measurable, and it is the observation point of the goal. Definite conclusions can be drawn through actual observation of the object. Generally speaking, the evaluation index system includes three levels of evaluation indexes: they are the relationship between gradual decomposition and refinement. Among them, the first-level evaluation indicators and the second-level evaluation indicators are relatively abstract and cannot be used as a direct basis for evaluation. The thirdlevel evaluation indicators should be specific, measurable, and behavior-oriented, and can be used as a direct basis for evaluation $[30,31]$.

3.4. Statistical Processing. Statistical analysis was performed with SPSS 13.0 statistical software. The significance test was performed by one-way analysis of variance, the difference between the two groups was performed by LSD- $t$ test, and the postoperative recovery of the thyroid was performed by group $t$ test. $P<0.05$ is considered significant and statistically significant.

\section{Mobile Medical Monitoring and Management System}

\subsection{System Test}

4.1.1. System Function Test. The functional of the system mainly includes eight functional modules including system login, control panel, GPS positioning module, Bluetooth module, data analysis module, data storage module, and data transmission module. The test results are shown in Table 2.

In the process of testing the monitoring effect, technical tests such as background sampling and encoding are carried out using specific video formats, and the server and other hardware are repeatedly tested until satisfactory test results are obtained. It can be seen from the table that the system is designed and tested successfully after analyzing the required functions.

4.1.2. System Performance Test. Here, we are mainly from five perspectives (response time, throughput, server resource occupancy, load test, and strength test) to carry out the performance test of the system, and the results are shown in Figure 4.

It can be seen from Figure 4 that the general response time is within $3 \mathrm{~s}$, and the user will feel more satisfied. 


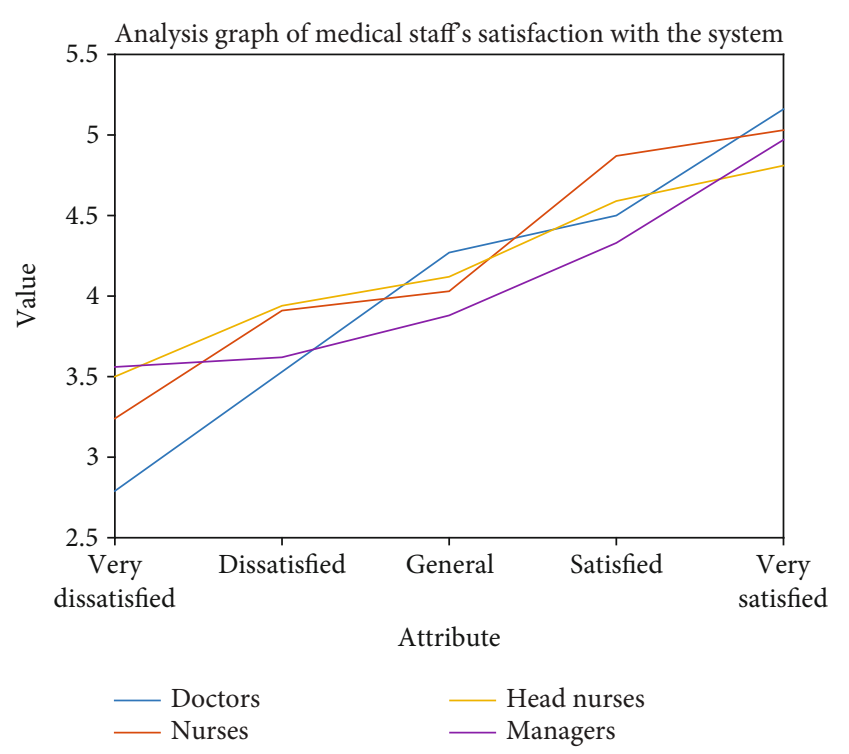

FIGURE 6: Analysis graph of medical staff's satisfaction with the system.

Between $3 \mathrm{~s}$ and $8 \mathrm{~s}$, users can barely accept it, and users who are longer than $8 \mathrm{~s}$ may not accept it. Because the cloud computing medical monitoring and management system is used to obtain the exact location of the patient in the first time, the response time needs to be shorter, and the response of this system within the time increases as the number of sensors increases $(P<0.05)$, but it is always much less than $3 \mathrm{~s}$; the throughput and server resource occupancy test scores do not rise much within 100 sensors; load test and strength test scores are at 200. The rise within the sensor is not large.

\subsection{Based on Monitoring System}

4.2.1. Analysis Based on the Energy Consumption of the Monitoring System. Here, the energy consumption of cloud computing, medical monitoring management system, and general monitoring system is compared, and the results are shown in Table 3.

It can be seen from Table 3 that the power consumption of the general monitoring system is not too high, but the cloud computing, medical monitoring, and management system, data is transmitted by sensors, and the power consumption is less than that of the general monitoring system; the equipment cost is relative to the general there are also fewer monitoring systems; the standby time of the two monitoring systems is very long; and the cloud computing medical monitoring management system has a wider range of action than the general monitoring system.

4.2.2. Analysis Based on the Transmission Speed of the Monitoring System. Here, we compare the energy consumption of the cloud computing medical monitoring and management system with the general monitoring system, and the results are shown in Table 4.

Table 4 shows that medical cloud computing monitoring and management system data uses visualization technology to visualize normal data, while the general tracking system is propagated by image transmission, so medical computing and cloud computing management system is relevant to the general, and the monitoring system is faster in data transmission speed. The cloud computing medical monitoring and management system directly detects the patient's heart rate, blood pressure, and other physiological data in real-time. Compared to the general monitoring system, it can only read the patient image. To be precise, stronger in the ability to process information and more help to medical staff.

\subsubsection{Analyze the Adverse Reactions of Patients after Surgery} Based on the Monitoring System. It can be seen from Figure 5 that the monitoring system can detect the adverse reactions of the patients based on the patient's physiological data. A total of $25 \%$ of patients have postoperative complications, including $8.33 \%$ of thyroid crisis, $8.33 \%$ of hand and foot convulsions, with $4.17 \%$ bleeding, and $4.17 \%$ hypocalcemia, for medical emergency and medical care, obtaining the exact location of the patient at the first time will bring a lot of time to the success of the rescue.

4.3. System Satisfaction Based on Medical Staff. It can be seen from Figure 6 that doctors' satisfaction with cloud computing, medical monitoring, and management system increased from 2.79 to 5.16; nurses' satisfaction with cloud computing, medical monitoring, and management system increased from 3.24 to 5.03; head nurses' satisfaction with cloud computing, medical monitoring, and management system the degree of satisfaction increased from 3.50 to 4.81; the administrator's degree of satisfaction with the cloud computing medical monitoring and management system increased from 3.56 to 4.97 , which shows that the medical staff are very satisfied with this system.

\section{Conclusions}

The research of mobile medical monitoring and management system started relatively late. At present, a complete monitoring system has been built on many large-scale structures at home and abroad to ensure the normal operation of the structure during service. The Internet of Things and cloud computing technologies, as new technologies developed in recent years, have high research and application value and can play a major role in promoting economic development and industrial upgrading. Analyzing the characteristics of the Internet of Things, cloud computing technology, and mobile medical monitoring, comparing it from three levels, and demonstrating the functional correspondence between their system architectures and the three common features: comprehensive perception, reliable transmission, and intelligent processing. Mobile medical monitoring is a specific application of the Internet of Things and cloud computing technology in the field of engineering monitoring. For medical emergency and medical care, obtaining the exact location of the patient for the first time is of great significance to the success of the rescue. This article implements the function of sending the location of the patient to the designated medical caregiver in the map service. It provides an effective way 
for patients to get help as soon as they encounter emergencies.

\section{Data Availability}

The data underlying the results presented in the study are available within the manuscript.

\section{Conflicts of Interest}

The authors declare that they have no conflicts of interest.

\section{Acknowledgments}

This work was supported by the Natural Science Foundation of China under Grant No. 61802267, by the Shenzhen Municipal Science and Technology Innovation Council under (Grant Nos. JCYJ20190813100801664 and JCYJ20160429182058044), and in part by Science and Technology talent program of Shenzhen University General Hospital Grant No. SUGH2018QD051.

\section{References}

[1] O. Edafe, E. Cochrane, and S. P. Balasubramanian, "Reoperation for bleeding after thyroid and parathyroid surgery: incidence, risk factors, prevention, and management," World Journal of Surgery, vol. 44, no. 4, pp. 1156-1162, 2020.

[2] K. G. Srinivasa, B. J. Sowmya, A. Shikhar, R. Utkarsha, and A. Singh, "Data analytics assisted internet of things towards building intelligent healthcare monitoring systems," Journal of Organizational and End User Computing, vol. 30, no. 4, pp. 83-103, 2018.

[3] T. von Ahnen, M. von Ahnen, S. Militz et al., "Compartment pressure monitoring after thyroid surgery: a possible method to detect a rebleeding," World Journal of Surgery, vol. 41, no. 9, pp. 2290-2297, 2017.

[4] B. C. Brajcich and C. R. Mchenry, "The utility of intraoperative nerve monitoring during thyroid surgery," Journal of Surgical Research, vol. 204, no. 1, pp. 29-33, 2016.

[5] H. Hamidi and M. Jahanshahifard, "The role of the internet of things in the improvement and expansion of business," Journal of Organizational and End User Computing, vol. 30, no. 3, pp. 24-44, 2018.

[6] I. H. Gardner, G. M. Doherty, and D. McAneny, "Intraoperative nerve monitoring during thyroid surgery," Current Opinion in Endocrinology, Diabetes, and Obesity, vol. 23, no. 5, pp. 394-399, 2016.

[7] R. Malik and D. Linos, "Intraoperative neuromonitoring in thyroid surgery: a systematic review," World Journal of Surgery, vol. 40, no. 8, pp. 2051-2058, 2016.

[8] K. V. Chávez, J. Ramírez, J. P. Pantoja, M. Sierra, D. Velázquez-Fernández, and M. F. Herrera, "Continuous intraoperative neural monitoring in thyroid surgery: a Mexican experience," Updates in Surgery, vol. 69, no. 4, pp. 505508, 2017.

[9] A. Jansen, J. P. Berg, O. Klungsøyr, M. H. B. Müller, J. L. Lyche, and J. O. Aaseth, "The influence of persistent organic pollutants on thyroidal, reproductive and adrenal hormones after bariatric surgery," Obesity Surgery, vol. 30, no. 4, pp. 13681378, 2020.
[10] R. Schneider, G. Randolph, G. Dionigi et al., "Prospective study of vocal fold function after loss of the neuromonitoring signal in thyroid surgery: the International Neural Monitoring Study Group's POLT study," The Laryngoscope, vol. 126, no. 5, pp. 1260-1266, 2016.

[11] J. Scharpf and G. Randolph, "Intraoperative nerve monitoring for parathyroid surgery," in Medical and Surgical Treatment of Parathyroid Diseases, B. Stack Jr. and D. Bodenner, Eds., pp. 459-468, Springer, 2017.

[12] M. M. Chen, R. K. Orosco, G. C. Lim, and F. C. Holsinger, "Improved transoral dissection of the tongue base with a next-generation robotic surgical system," The Laryngoscope, vol. 128, no. 1, pp. 78-83, 2018.

[13] N. Komune, K. Matsushima, S. Matsuo, S. Safavi-Abbasi, N. Matsumoto, and A. L. Rhoton, "The accuracy of an electromagnetic navigation system in lateral skull base approaches," The Laryngoscope, vol. 127, no. 2, pp. 450-459, 2017.

[14] S. R. Chandra and Y. Wang, "Cloud things construction-the integration of internet of things and cloud computing," Future Generation Computer Systems, vol. 56, pp. 684-700, 2016.

[15] M. Díaz, C. Martín, and B. Rubio, "State-of-the-art, challenges, and open issues in the integration of Internet of things and cloud computing," Journal of Network and Computer Applications, vol. 67, pp. 99-117, 2016.

[16] M. Nazari Jahantigh, A. Masoud Rahmani, N. Jafari Navimirour, and A. Rezaee, "Integration of internet of things and cloud computing: a systematic survey," IET Communications, vol. 14, no. 2, pp. 165-176, 2020.

[17] A. Kobusińska, C. Leung, C. H. Hsu, S. Raghavendra, and V. Chang, "Emerging trends, issues and challenges in Internet of Things, Big Data and cloud computing," Future Generation Computer Systems, vol. 87, pp. 416-419, 2018.

[18] A. Celesti, O. Amft, and M. Villari, "Guest editorial special section on cloud computing, edge computing, internet of things, and big data analytics applications for healthcare industry 4.0," IEEE Transactions on Industrial Informatics, vol. 15, no. 1, pp. 454-456, 2019.

[19] J. Yang, C. Wang, Q. Zhao, B. Jiang, Z. Lv, and A. K. Sangaiah, "Marine surveying and mapping system based on Cloud Computing and Internet of Things," Future Generation Computer Systems, vol. 85, pp. 39-50, 2018.

[20] P. N. Mahalle, M. S. Pathan, and V. V. Kimbahune, "Special issue on internet of things, next generation networks, data mining, and cloud computing 2017- part II," International Journal of Synthetic Emotions, vol. 8, no. 2, pp. 76-76, 2017.

[21] J. Dizdarević, F. Carpio, A. Jukan, and X. Masip-Bruin, “A survey of communication protocols for internet of things and related challenges of fog and cloud computing integration," ACM Computing Surveys, vol. 51, no. 6, pp. 1-29, 2018.

[22] W. Rivera, Sustainable Cloud and Energy Services |Cloud Computing and Internet of Things Integration: Architecture, Applications, Issues, and Challenges, Springer, 2018.

[23] B. d. T. P. Gomes, L. C. M. Muniz, F. J. da Silva e Silva, L. E. T. Ríos, and M. Endler, "A comprehensive and scalable middleware for ambient assisted living based on cloud computing and internet of things," Concurrency and Computation: Practice and Experience, vol. 29, no. 11, article e4043, 2017.

[24] F. Wu, X. Li, L. Xu, A. K. Sangaiah, and J. J. P. C. Rodrigues, "Authentication protocol for distributed cloud computing: an explanation of the security situations for internet-of-things- 
enabled devices," IEEE Consumer Electronics Magazine, vol. 7, no. 6, pp. 38-44, 2018.

[25] R. Deng, R. Lu, C. Lai, T. H. Luan, and H. Liang, "Optimal workload allocation in fog-cloud computing toward balanced delay and power consumption," IEEE Internet of Things Journal, vol. 3, no. 6, pp. 1171-1181, 2017.

[26] F. Xiao and W. Ding, "Divergence measure of Pythagorean fuzzy sets and its application in medical diagnosis," Applied Soft Computing, vol. 79, pp. 254-267, 2019.

[27] X. Li, H. Jianmin, B. Hou, and P. Zhang, "Exploring the innovation modes and evolution of the cloud-based service using the activity theory on the basis of big data," Cluster Computing, vol. 21, no. 1, pp. 907-922, 2018.

[28] Z. Lv and F. Piccialli, "The security of medical data on internet based on differential privacy technology," ACM Transactions on Internet Technology (TOIT), vol. 21, no. 3, pp. 1-18, 2021.

[29] S. Wan, Z. Gu, and Q. Ni, "Cognitive computing and wireless communications on the edge for healthcare service robots," Computer Communications, vol. 149, pp. 99-106, 2020.

[30] S. Biswas, D. Devi, and M. Chakraborty, "A hybrid case based reasoning model for classification in internet of things (Iot) environment," Journal of Organizational and End User Computing, vol. 30, no. 4, pp. 104-122, 2018.

[31] M. Elhoseny, G.-B. Bian, S. K. Lakshmanaprabu, K. Shankar, A. K. Singh, and W. Wu, "Effective features to classify ovarian cancer data in internet of medical things," Computer Networks, vol. 159, pp. 147-156, 2019. 